\title{
Load Dependent Frictional Response of Vertically Aligned Single-Walled Carbon Nanotube Films
}

\author{
Andrew S. Westover, ${ }^{\text {a,b }}$, Junho Choi ${ }^{c}$, Kehang Cui ${ }^{\mathrm{c}}$, Takumi Ishikawa ${ }^{\mathrm{c}}$, Taiki Inoue ${ }^{\mathrm{c}}$, Rong Xiang ${ }^{\mathrm{c}}$, \\ Shohei Chiashi ${ }^{\mathrm{c}}$, Takahisa Kato ${ }^{\mathrm{c}}$, Shigeo Maruyama, ${ }^{\mathrm{c},{ }^{*}}$, and Cary L. Pint ${ }^{1,2, *}$ \\ anterdisciplinary Materials Science and Engineering Program, Vanderbilt University, Nashville, TN 37212, USA \\ ${ }^{\mathrm{b}}$ Mechanical Engineering Department, Vanderbilt University, Nashville, TN 37212, USA \\ ${ }^{c}$ Department of Mechanical Engineering, The University of Tokyo, Tokyo 113-8656, JAPAN \\ d Energy NanoEngineering Lab., National Institute of Advanced Industrial Science and Technology, Tsukuba 305-8564, \\ JAPAN \\ *Authors to whom correspondence should be addressed. Electronic mail: cary.l.pint@vanderbilt.edu, \\ maruyama@photon.t.u-tokyo.ac.jp
}

\begin{abstract}
Here we use microscratch testing to demonstrate how a single-walled carbon nanotube (SWCNT) forest material can exhibit variable adhesion properties with solid surfaces ranging from negligible adhesion at low loading due to the normal alignment of SWCNTs to maximum adhesion at high loading that exploits the extraordinary sidewall adhesion of SWCNTs. This observation, which exhibits no analog in conventional bulk materials, is correlated to loading-induced structural modification of the low-density SWCNT-substrate interface morphology. This observation opens new pathways to use structural modification of low density materials to engineer and control a wide range of adhesion properties with solid surfaces.
\end{abstract}

Keywords: Scratch Test, Single Walled Carbon Nanotubes, Friction, Adhesion, Low-Density Materials

(C) 2016. This manuscript version is made available under the Elsevier user license http://www.elsevier.com/open-access/userlicense/1.0/ 
Nanostructured material interfaces with solid surfaces represent a platform for technologies ranging from batteries, flexible electronics, and solar cells, to transparent conductive thin films.[1-3] Despite the widespread application of these systems, the interfacial mechanics associated with such nanostructured material interfaces remains poorly understood.[4-6] Building from a vast body of literature on the mechanical properties of interfaces of inorganic and organic films, characteristics such as the microstructure, crystal structure, grain size, material homogeneity, and defects[7] in inorganic films and crystallinity, polymer chain length, chain orientation, and plasticizer content[8-10] in organic films can describe the film-dependent mechanical properties. In contrast, nanomaterial interfaces exhibit mechanical properties entirely distinct from these previous studies that arise from characteristics such as nanomaterial density, nanomaterial morphology, and individual chemical/physical properties of the nanomaterials.[11-13] Whereas mechanical behavior of individual nanostructures is a dynamic area of research due to observations of superlubrication and other phenomena at the nanoscale, studies extending fundamental ideas observed at single-particle scales to complex film assemblies are only starting to recently emerge.[14-18]

Of particular interest to such applications are carbon nanotubes, which are readily grown or processed into complex networks that can exhibit unique frictional characteristics, such as strong shear-on binding response up to $100 \mathrm{~N} / \mathrm{cm}^{2}$ and easy normal lift off.[19-21] These unique carbon nanotube networks have been shown to exhibit frictional properties 10X improved from a natural gecko foot, and further enable contact transfer of complex stacks of organized carbon nanotubes to arbitrary substrates.[22, 23] Other recent advances have focused on in-situ TEM and SEM compression and tensile testing of nanomaterial pillars and films,[12, 24, 25] and nanoindentation of CNT films to understand and engineer the hardness and compressive behavior.[13, 26] Whereas early studies have indicated the ability to apply scratch testing approaches to complex CNT-based networks, $[27,28]$ studies intersecting the exciting applications of the CNT-based frictional characteristics, such as forming functional adhesives, and more conventional techniques for testing mechanical properties of inorganic and organic film interfaces remain mostly unexplored. 
Here we adapt a microscratch technique traditionally used to determine the adhesion of thin films to substrates[29, 30] to study the load-dependent frictional response of vertically aligned single-walled carbon nanotube (SWCNT) thin films grown by alcohol-assisted catalytic chemical vapor deposition (ACCVD). [31-33] Our experiments indicate a load-dependent frictional response where coupling of mechanical energy into the SWCNT material modifies the interfacial morphology of the SWCNTs and leads to increased adhesion properties until reaching a load threshold where maximum frictional response is obtained. Our work highlights how external loading can be used to actuate frictional properties in low density networks of SWCNTs in a manner generalizable to other low density materials composed of nanostructured building blocks.

SWCNT films were synthesized using ACCVD as detailed in the supplementary information. Microscratch tests in this study were performed using a RHESCA CSR-02 microscratch testing system with a $100 \mu \mathrm{m}$ diameter diamond tipped stylus. The testing apparatus (Fig.1) consists of a traditional audio cartridge typically used for record players, where a magnetic stylus is connected to a magnetic sensing coil. The scratch tests involve two motions in a single $x-y$ plane: (1) an oscillatory motion in the $x$-direction described by $X=X_{0} \operatorname{Cos}(\omega t)$ (Fig. 1a), and (2) a linear lateral motion normal to the oscillatory motion (y-direction). To induce a constantly increasing force on the SWCNT film, the film was maintained at a slight angle of $1^{\circ}$ relative to the lateral motion in the $y$ direction (Fig. 1b). With this angle and tip the microscratch measurement has a loading rate of $17.64 \mu \mathrm{N} / \mu \mathrm{m}$ in the $\mathrm{y}$-direction of motion while oscillatory motion in the $\mathrm{x}$-direction enables the measurement of friction. In this system, the frictional force between the stylus and the film causes the stylus to lag behind the cartridge motion, generating a voltage response. This voltage response is proportional to the frictional response of the thin film, and is conventionally represented in units of $\mathrm{V}$ or in arbitrary units due to known calibration challenges.[34] Following fast Fourier transform (FFT) noise correction, the measured response is proportional to the friction force measured by the stylus. In a traditional microscratch measurement on a conventional thin film, initially the stylus is maintained at a z-distance above the thin film and there is an initial period with no measured response. Once the stylus engages the thin film through movement in the y-axis direction, mechanical energy is coupled into the film with the normal component of the applied load proportional to the displacement, and a linearly increasing frictional response is observed until the thin film detaches from the substrate at a critical load which 
corresponds to a rapid jump in the frictional response followed by large fluctuations (see Fig. 1c).[29] A comparison of the raw signal and the FFT signal for the data in the main text is presented in Fig. S1. Notably, the microscratch data in Fig. 1c is representative of a measurement using this technique from a conventional coating, and only included for a generalized comparison between a conventional scratch test and our microscratch data on a SWCNT film.

Fig. 2 shows three microscratch experiments on a SWCNT film showing the measured frictional response of the SWCNT film as a function of displacement (bottom) and applied load (top). By comparing the measured frictional response with the traces of the scratches (Fig. S2), three general regimes are identified. The first regime is highlighted in blue in Fig. 2, and corresponds to a range of displacements where the stylus had engaged the SWCNT film and removed it from the surface without any measureable frictional response (from 0 to $0.6 \mathrm{mN}$ ). We attribute this regime to the weak normal binding of SWCNTs oriented perpendicular to a solid substrate in a low density SWCNT material. With increasing lateral displacement in the y-direction, a second regime highlighted in red is encountered where both the stylus is engaged with the SWCNT film and a small frictional response is recorded (from 0.6 to $2.3 \mathrm{mN}$ ). The onset of this regime $(\sim 0.6 \mathrm{mN})$ represents the point where external loading starts to disrupt the normal orientation of SWCNTs at the SWCNT-substrate interface, and increased adhesion is measured. Finally, a third regime is highlighted in gold where a greater slope is observed in the frictional response indicating a significantly enhanced coefficient of friction (from $2.3 \mathrm{mN}$ to $5.7 \mathrm{mN}$ ). At the very end of this regime, a maximum value of the frictional response is achieved at a point attributed to a condition where increasing mechanical energy leads to no further increase in the frictional response. With exception of minor variance between measurements, it is notable that this same pattern was measured in all three of the microscratch experiments shown in Fig.2. Beyond the point of maximum frictional response from the CNT film, a stick-slip response is measured that is attributed to the convoluted frictional response between the stylus, the SWCNT film, and the underlying substrate.

Closer examination of the SEM trace from the scratches (Fig. 3 and Fig. S2) indicates full removal of the SWCNT film in the first regime, with only partial removal of the SWCNT film in the subsequent regimes. Removal of the film indicates that the adhesion of the SWCNT film to the stylus is greater than to the substrate. 
As more of the CNT film remain after the microscratch tests when a greater force is applied to the substrate, we can deduce that the SWCNT film has increased adhesion to the substrate when greater forces are applied to the substrate. In order to better understand the measured frictional response, SEM imaging was performed of the SWCNT films remaining following the scratch tests, and reveals a distinct change in the morphology of the film as a load is applied. Initially the SWCNT film is in a low-density vertically aligned architecture (Fig. 3b), which becomes partially collapsed into a randomly aligned network (Fig. 3c) in the second regime. Finally in the third regime, the packing of the SWCNTs on the surface is too dense to resolve individual SWCNT bundles in the SEM images (Fig. 3d). Building from previous studies,[22] this is correlated to the formation of a highly dense and semi-aligned SWCNT material due to the applied load.

These results indicate that the applied load from the stylus is coupled into mechanical energy that modifies the morphological properties of the SWCNT material, and the SWCNT-substrate interface. This is attributed to the observed load-dependent frictional response. Previous studies have highlighted the strong sidewall adhesion of SWCNT materials with surfaces that enable exceptional adhesion capability for CNT-based gecko tapes.[19-21] However, our work not only emphasizes the importance of sidewall adhesion, but the application of controlled loading to maximize the density of SWCNT-substrate interfacial contact sites. At the very end of the third regime in Fig. 2, the maximum loading is achieved at a point where further coupling of mechanical energy into the SWCNT material leads to no further modification of the frictional properties, and hence no further morphological change of SWCNT at the solid surface.

To better understand this we calculated the areal packing fraction, or the percentage of a surface cross section occupied by SWCNTs (details in the supplementary information) as a function of lateral displacement and applied load (Fig. 4a -black) and compared this to a traditional thin film (Fig. 4a - red) where the entire surface is exposed to the stylus resulting in an areal packing fraction of approximately $100 \%$. As the load is increased on the SWCNT film, the confined space between the stylus and the substrate causes the SWCNT to be compressed thus exposing more of the individual SWCNT side walls to interact with the stylus. The correlation between the curve presented in Fig. 4 and the experimental data in Fig. $2 \& 3$ indicates that the increasing coefficient of friction observed as the load increases on the SWCNT film is likely explained by the restructuring of the SWCNT film 
with an applied load as illustrated in Fig. 4b. Notably this response differs significantly from a traditional thin film. Whereas a traditional thin film typically consists of a bulk material without void space, increasing loads cause elastic compression of the film (Fig. 4c). This typically results in a frictional response similar to the simulated response in Fig. 1c, and does not typically exhibit a significant change in the coefficient of friction until the critical load is reached and the thin film detaches from the substrate.

A key outcome of this analysis is the observation that the maximum frictional response of a carbon nanotube adhesive is a function of the maximum areal packing fraction that can be achieved by coupling of mechanical energy into the material. This opens routes to understand not only interface properties of CNT materials, but also the response of CNT assemblies with different characteristics such as alignment, density, diameter, and surface treatment that will directly impact the frictional characteristics of the surfaces. This type of morphologydependent frictional response is unique to a low-density nanostructured network and is significantly distinguished from that obtained in conventional bulk coatings or films of organic or inorganic materials.

In summary, our study reports the use of microscratch analysis to elucidate the load-dependent frictional response of aligned SWCNT materials. Unlike traditional coatings, we observe that morphological changes at the SWCNT-substrate interface lead to an increasing frictional response as a load is applied due to the coupling of mechanical energy into the SWCNT material that leads to restructuring at the SWCNT-substrate interface. This occurs until a maximum frictional response is achieved, at which point no further mechanical energy can be coupled into the SWCNT array and the frictional response levels off. This work opens new exciting avenues for research in areas such as (1) the study of how network properties of SWCNTs or CNTs can modulate the maximum frictional response, since the total mechanical energy coupled into the material will be a function of the initial morphology, and (2) the study of how surface engineering, such as with atomic layer deposition, can simultaneously impact the elastic properties and interfacial frictional properties of similar low-density materials. The technique and results reported in this work opens a pathway for the advanced study and design of nextgeneration nanoscale adhesives as well as actuators that can widely modulate the frictional characteristics of surfaces using external stimuli.

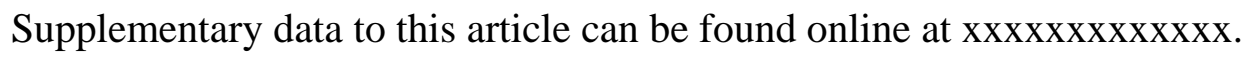


The authors thank Anna Douglas, Keith Share, and Landon Oakes for insightful discussions. This project was supported by the National Science Foundation East Asia and Pacific Summer Institute 2015 award number 1515541, and the Japan Society for the Promotion of Science's summer program 2015. A part of this work was financially supported by Grants-in-Aid for Scientific Research (15H02219, 15H05760) and IRENA Project by JST-EC DG RTD, Strategic International Collaborative Research Program, SICORP.

\section{References}

[1] A.L. Gorkina, A.P. Tsapenko, E.P. Gilshteyn, T.S. Koltsova, T.V. Larionova, A. Talyzin, A.S. Anisimov, I.V. Anoshkin, E.I. Kauppinen, O.V. Tolochko, Carbon 100 (2016) 501-507.

[2] R. Carter, L. Oakes, A.P. Cohn, J. Holzgrafe, H.F. Zarick, S. Chatterjee, R. Bardhan, C.L. Pint, J. Phys. Chem. C 118(35) (2014) 20137-20151.

[3] K. Cui, A.S. Anisimov, T. Chiba, S. Fujii, H. Kataura, A.G. Nasibulin, S. Chiashi, E.I. Kauppinen, S. Maruyama, J. Mater. Chem. A 2(29) (2014) 11311-11318.

[4] J. Xu, T.S. Fisher, Int. J. Heat Mass Transfer 49(9) (2006) 1658-1666.

[5] H.-C. Su, C.-H. Chen, Y.-C. Chen, D.-J. Yao, H. Chen, Y.-C. Chang, T.-R. Yew, Carbon 48(3) (2010) 805-812.

[6] Y. Maeno, A. Ishikawa, Y. Nakayama, Appl. Phys Express 3(6) (2010) 065102.

[7] D. Munz, T. Fett, Ceramics: mechanical properties, failure behaviour, materials selection, Springer Science \& Business Media, New York, 2013.

[8] I.M. Ward, J. Sweeney, Mechanical properties of solid polymers, John Wiley \& Sons, United Kingdom, 2012.

[9] E. Manias, A. Touny, L. Wu, K. Strawhecker, B. Lu, T. Chung, Chem. Mater. 13(10) (2001) 35163523. 
[10] A.S. Westover, F.N. Shabab, J.W. Tian, S. Bernath, L. Oakes, W.R. Erwin, R. Carter, R. Bardhan, C.L. Pint, J. Electrochem. Soc. 161(6) (2014) E112-E117.

[11] A. Baji, Y.-W. Mai, S.-C. Wong, M. Abtahi, P. Chen, Compos. Sci. Technol. 70(5) (2010) 703-718. [12] S.B. Hutchens, L.J. Hall, J.R. Greer, Adv. Funct. Mater. 20(14) (2010) 2338-2346.

[13] M.R. Maschmann, Q. Zhang, R. Wheeler, F. Du, L. Dai, J. Baur, ACS Appl. Mater. Interfaces 3(3) (2011) 648-653.

[14] C. Lee, Q. Li, W. Kalb, X.-Z. Liu, H. Berger, R.W. Carpick, J. Hone, Science 328(5974) (2010) 7680.

[15] J.C. Spear, B.W. Ewers, J.D. Batteas, Nano Today 10(3) (2015) 301-314.

[16] P. Egberts, G.H. Han, X.Z. Liu, A.C. Johnson, R.W. Carpick, ACS Nano 8(5) (2014) 5010-5021.

[17] Q. Li, X.-Z. Liu, S.-P. Kim, V.B. Shenoy, P.E. Sheehan, J.T. Robinson, R.W. Carpick, Nano Lett. 14(9) (2014) 5212-5217.

[18] J. Cumings, A. Zettl, Science 289(5479) (2000) 602-604.

[19] L. Qu, L. Dai, M. Stone, Z. Xia, Z.L. Wang, Science 322(5899) (2008) 238-242.

[20] S. Sethi, L. Ge, L. Ci, P.M. Ajayan, A. Dhinojwala, Nano Lett. 8(3) (2008) 822-825.

[21] L. Ge, S. Sethi, L. Ci, P.M. Ajayan, A. Dhinojwala, Proc. Natl. Acad. Sci. 104(26) (2007) 1079210795.

[22] C.L. Pint, Y.-Q. Xu, M. Pasquali, R.H. Hauge, ACS Nano 2(9) (2008) 1871-1878.

[23] C.L. Pint, Y.-Q. Xu, S. Moghazy, T. Cherukuri, N.T. Alvarez, E.H. Haroz, S. Mahzooni, S.K.

Doorn, J. Kono, M. Pasquali, ACS Nano 4(2) (2010) 1131-1145.

[24] S. Pathak, E.J. Lim, P. Pour Shahid Saeed Abadi, S. Graham, B.A. Cola, J.R. Greer, ACS Nano 6(3) (2012) 2189-2197.

[25] X. Zhang, K. Jiang, C. Feng, P. Liu, L. Zhang, J. Kong, T. Zhang, Q. Li, S. Fan, Adv. Mater. 18(12) (2006) 1505-1510. 
[26] W. Xue, T. Cui, Nanotechnology 18(14) (2007) 145709.

[27] I. Lahiri, D. Lahiri, S. Jin, A. Agarwal, W. Choi, ACS Nano 5(2) (2011) 780-787.

[28] P. Dickrell, S. Pal, G. Bourne, C. Muratore, A. Voevodin, P. Ajayan, L. Schadler, W. Sawyer, Tribol. Lett. 24(1) (2006) 85-90.

[29] J. Choi, J. Kim, S. Nakao, M. Ikeyama, T. Kato, Nucl. Instrum. Methods Phys. Res., Sect. B 257(1) (2007) 718-721.

[30] P. Lu, H. Gomez, X. Xiao, M. Lukitsch, D. Durham, A. Sachdeve, A. Kumar, K. Chou, Surf. Coat. Technol. 215 (2013) 272-279.

[31] R. Xiang, Z. Yang, Q. Zhang, G. Luo, W. Qian, F. Wei, M. Kadowaki, E. Einarsson, S. Maruyama, J. Phys. Chem. C 112(13) (2008) 4892-4896.

[32] K. Cui, T. Chiba, S. Omiya, T. Thurakitseree, P. Zhao, S. Fujii, H. Kataura, E. Einarsson, S. Chiashi, S. Maruyama, J. Phys. Chem. Lett. 4(15) (2013) 2571-2576.

[33] S. Maruyama, R. Kojima, Y. Miyauchi, S. Chiashi, M. Kohno, Chem. Phys. Lett. 360(3) (2002) 229-234.

[34] S. Ogawa, Y. Babai, Y. Masashi, T.N. Akano, Adhesion Aspects of Thin Films, volume 2: Adhesion Aspects of Thin Films, CRC Press, Boca Raton, Florida, 2005, p. 203. 
(a)

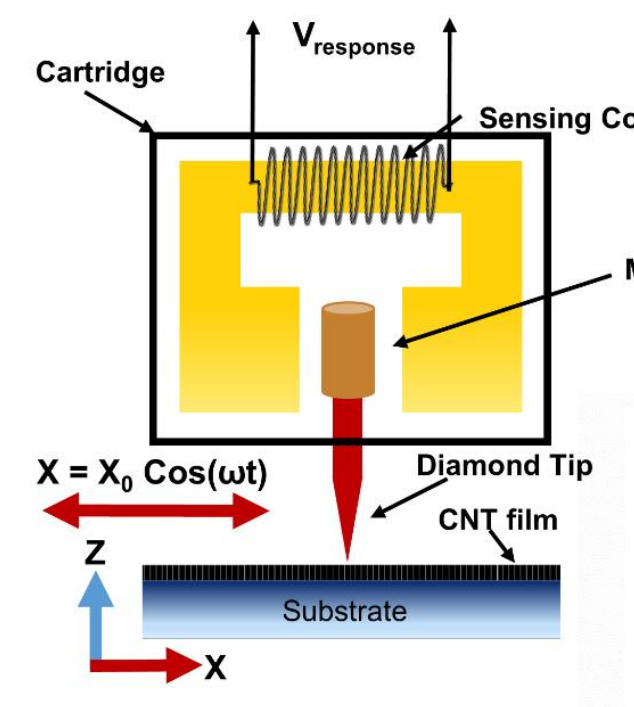

(b)



(c)



Fig. 1. Microscratch testing approach used for this study with the experimental apparatus and oscillatory motion depicted in (a) and the lateral motion depicted in (b) with an inset SEM image of the alcohol catalytic CVD grown SWCNT film tested. (c) A typical response using this technique with a conventional thin film coating. 


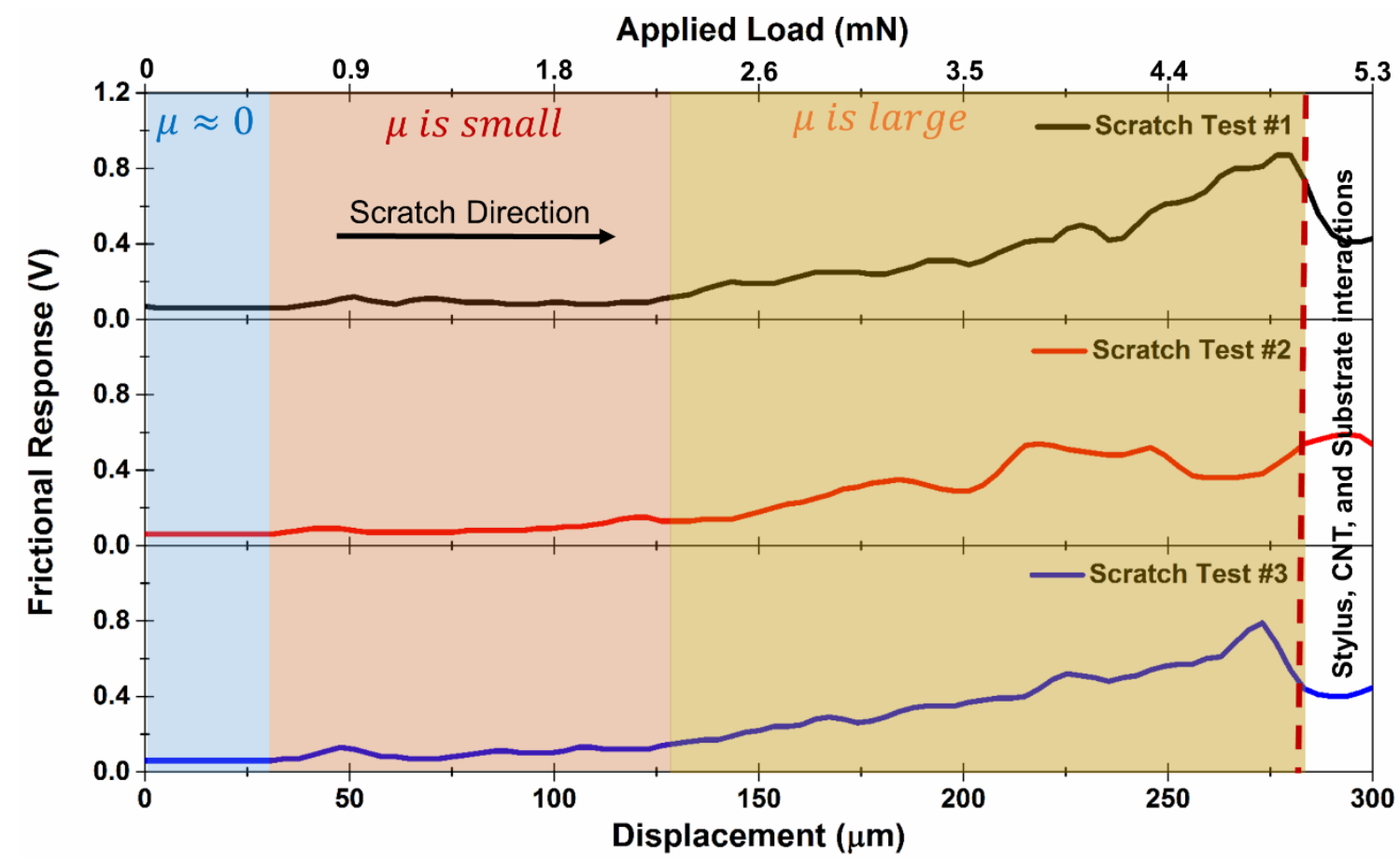

Fig. 2. (a) Three microscratch measurements showing the frictional response as a function of displacement(bottom) and applied load(top) on the same vertically aligned SWCNT material. The different frictional regimes discussed in the text are noted. 


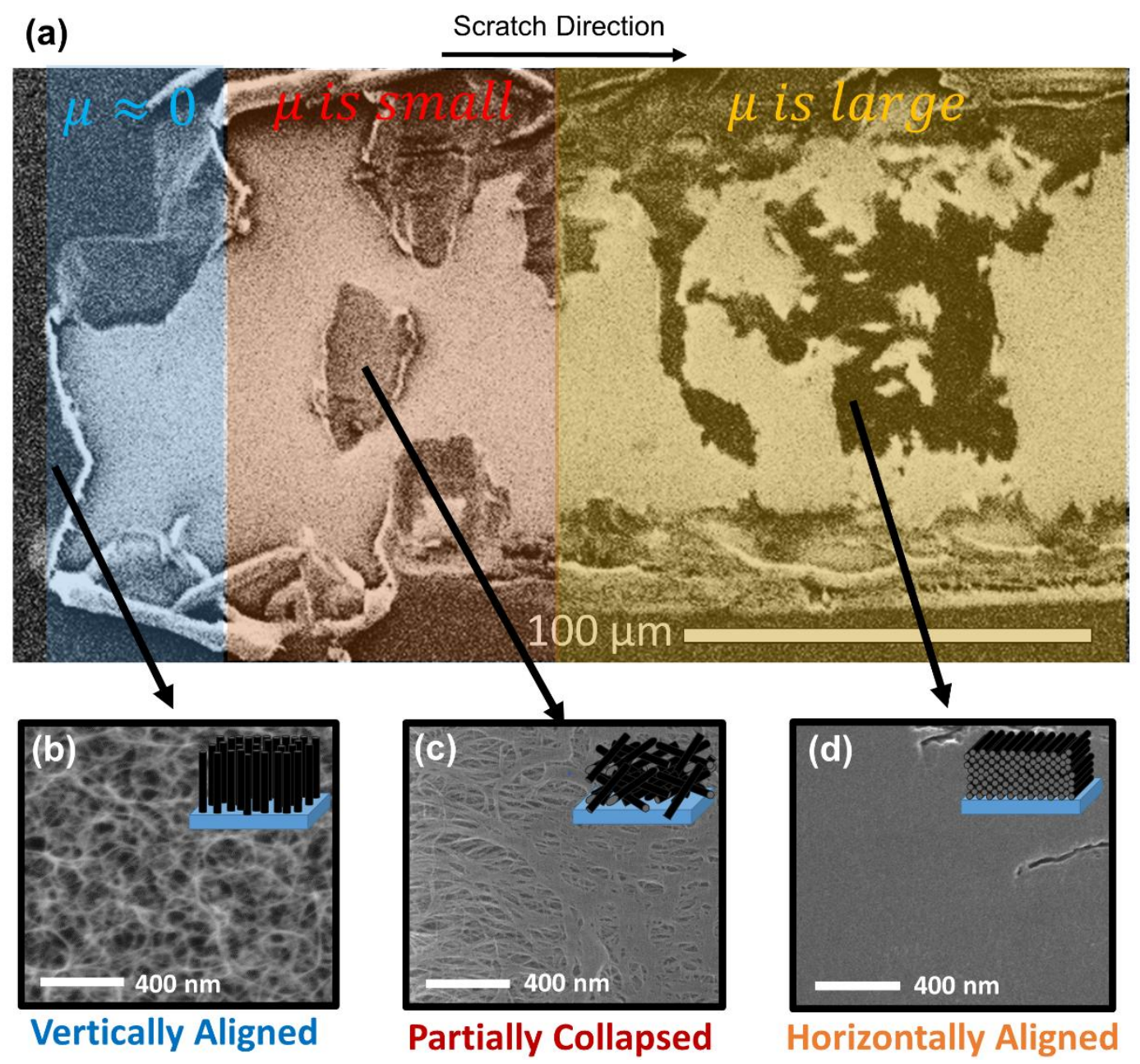

Fig. 3. (a) SEM image of the trace of one of the microscratch measurements depicted in Fig. 2 (scratch test \#3). (b-d) correspond to SEM images of the morphology of the CNT film as load is applied starting in the initial low-density aligned forest (b) changing to a partially collapsed SWCNT morphology (c) and finally reaching a high density horizontally aligned morphology (d). Notably, SEM images contain regions with CNT and without CNT. Whereas the presence of these regions do not influence the microscratch test, CNTs remain in some areas due to greater adhesion to the substrate versus the stylus during the experiment. 


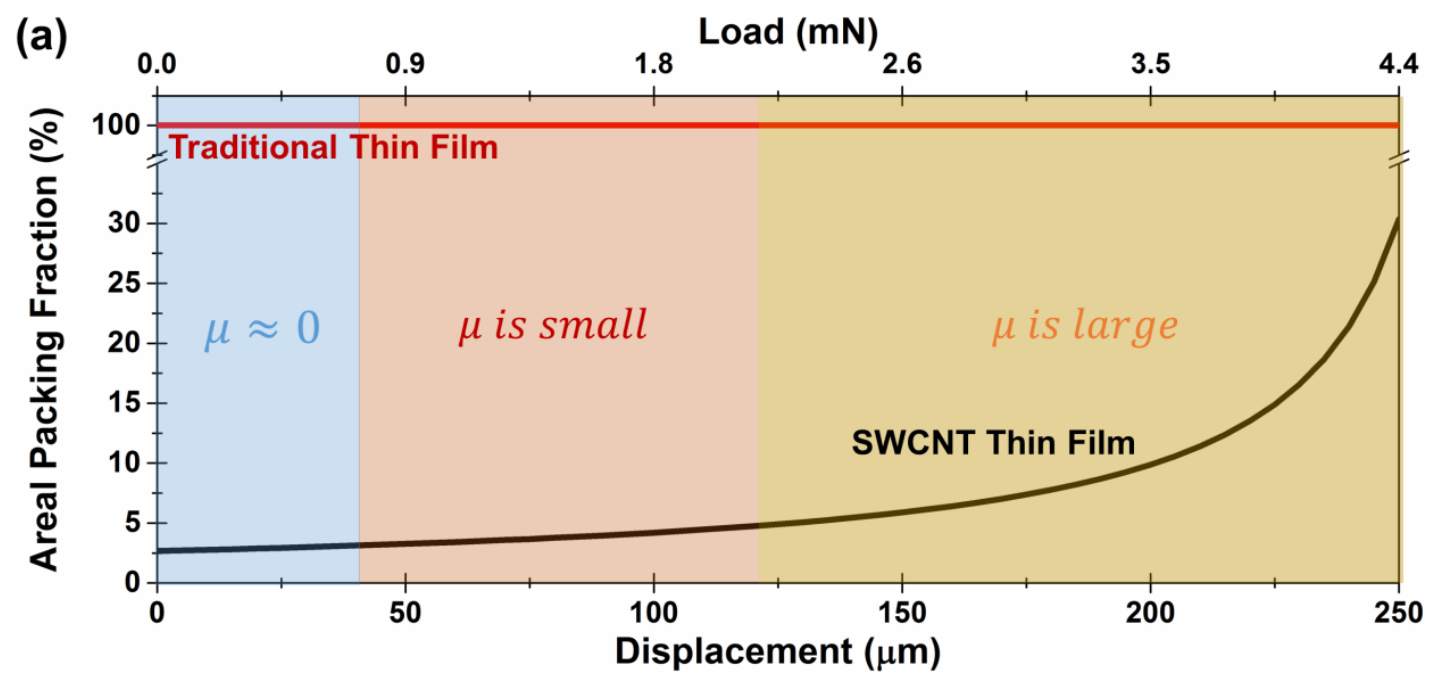

(b) SWCNT Thin Film - Plastic Deformation

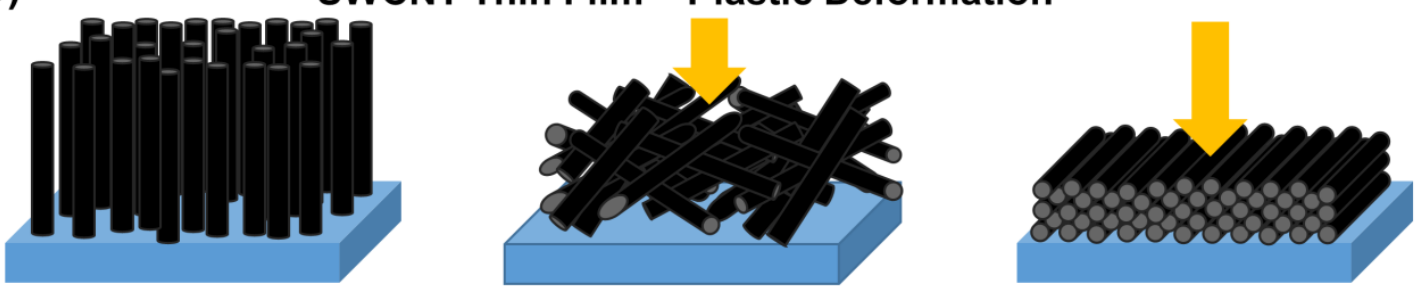

(c) Traditional Thin Film - Elastic Deformation
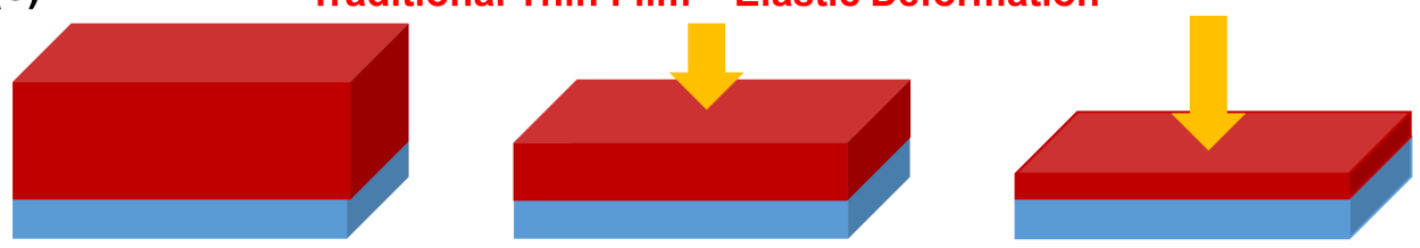

Fig. 4. (a) Calculations of estimated areal packing fraction of the SWCNT as a function of discplacement (bottom) and applied load (top) and compared to a traditional (bulk-like) thin film. This curve is consistent with the shape of the frictional response in Fig. 2, supporting interfacial morphology change as a mechanism of the frictional response. (b) Scheme illustrating the change in morphology of the SWCNT film with increasing load contrasted with the elastic response of a traditional thin film (c). 


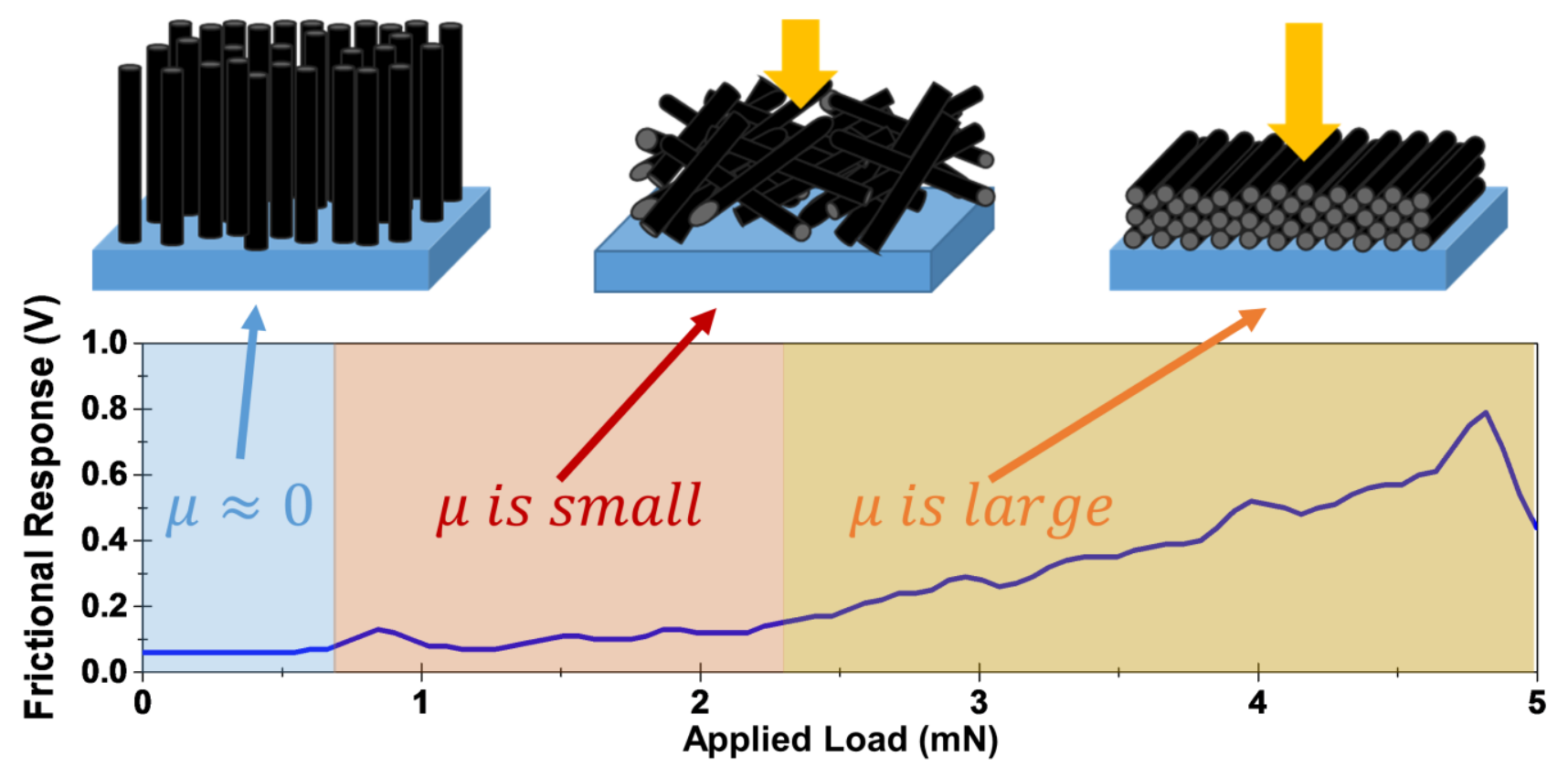

